anderseits die neue Gattung hinwieder der auch in Neu-Guinea vertretenen Gattung Gonocaryum Miq. Von dieser ist sie aber sehr verschieden hinsichtlich des Blütenstandes und besonders des Griffels. Sie scheint sich aber dennoch an letztere Gattung am engsten anzuschliessen.

Neu-Guinea: Kaiser-Wilhelms-Land, in den Wäldern des Gornadjidji, um $150 \mathrm{~m}$ (blühend am 23. Juni 1909. - Schlechter no. 19926).

\title{
LV. Friedrich Fedde, Neue Arten aus der Verwandtschaft der Corydalis aurea Willd. von Nord-Amerika.
}

(Originaldiagnosen.)

Bei der Durcharbeitung des Berliner Materials von Corydalis gelang es mir trotz seiner Spärlichkeit festzustellen, dass das Meiste, was in Nordamerika aus der Gattung Corydalis $\S$ Capnoïdes gesammelt wird, zwar als $C$, aurea Willd bestimmt ist, indessen aber doch ganz verschiedenen Arten angehören dürfte. Ich ging von den Originalexemplaren Will denows für Corydalis aurea und Fendlers bzw. Engelmanns für $C$. montana (C. aurea var. occidentalis) aus und konnte folgende neue Arten feststellen:

1. Corydalis Geyeri Fedde, spec. nov.

Herba, ut videtur, annua, glauco-viridis, humilis, a basi ramosa, caulibus brevibus diffusis striato-angulatis, $7,5-12 \mathrm{~cm}$ altis. Folia basalia satis multa rosulata et graciliter petiolata, caulina brevius petiolata, pinnatipartita, segmentis pinnatisectis, lobis integris vel lobulatis, ultimis plerumque trilobulatis, lineari-lanceolatis vel lanceolato-obcuneatis, minutis, apicem versus breviter acutis, petiolis ad basim vaginanter dilatatis. Flores in racemos terminales breves, paucifloros, primum confertos, deinde valde laxos, breviter pedunculatos dispositi, flavidi; bracteae pro rata magnae, 5-6 mm longae, latiuscule lanceolatae, acuminatae vel subapiculatae, pedicellos florum superantes, fructuum aequantes vel paullo breviores, integerrimae, infimae saepe pinnatim incisae. Sepala valde diminuta, circiter $0,05 \mathrm{~cm}$ longa, squamulaeformia, membranacea, subtrianguliformia, leviter grosse serrulata, permox decidentia; petalum superius carinatum, ad lobos valleculato-repandum, non mucronulatum, carina apicem versus leviter geniculata, ad dorsum crista brevi, sed alta, leviter repanda, apicem non attingente, sed ibi quasi oblique truncata, retro breviter rotundata ornata, $0,7-0,8 \mathrm{~cm}$ longum, calcari vix quartam partem totius petali aequante, leviter decurvato, bullato-rotundato; petalum inferius leviter carinatum, non mucronulatum, carina leviter geniculata, ad dorsum crista subsemilunata, leviter denticulatorepanda, brevi ornata; petala interiora unguiculata, ungue linearioblongo, lamina obliqua, versus petalum superius truncato-lobata, versus inferiorem paulatim attenuata, vix subpanduraeformia, ad dorsum crista 
brevi, at alta, oblique semilunata, apicem laminae non attingente, sed ibi quasi oblique truncata, retro basim laminae non attingente ornata; staminum superiorum appendix nectariferus brevis et curvatus. Siliqua (immatura) patens vel subpendula, linearis, teres (?), leviter torulosa, recta vel subarcuata, pedunculo gracili quam siliqua breviore, 1,25$1,5 \mathrm{~cm}$ longa, stylo acuminata.

Gebiet des atlantischen Nord-Amerika; Provinz des sommergrünen Mississippi-Waldes. - Missouri: „Loose suil. fertile woods", St. Louis (Ch. A. Geyer, fl. fr., V, 1842 sub C. aurea!)

Nota: Praeclara habitu humili, sepalis valde diminutis squamulaeformibus; a $C$. aurea crista petalorum exteriorum prorsus diversa.

2. Corydalis wyomingensis Fedde, spec. nov.

Herba biennis vel perennis, radice sublignosa cortice obscure brunea obtecta, glauca, magis erecta quam humilis, a basi ramosa, caulibus magis erectis quam diffusis, striato-angulatis, $20-30 \mathrm{~cm}$ alta. Folia alterna, petiolata, bipinnatipartita, lobis pinnatilobatis, lobulis, ultimo plerumque trifido, lanceolatis vel anguste obovato-lanceolatis, breviter acutis, intermediis ultimis paullo latioribus submucronulatis, petiolis ad basim non ita vaginanter dilatatis. Flores flavidi in racemos terminales et laterales, 6-12-floros, primum confertos, deinde paullo laxiores, subsecundos, breves, folia non superantes, non ita longe pedunculatos dispositi; bracteae lineari-lanceolatae vel lanceolatae, imprimis ad apicem saepius leviter denticulatae graciliter acuminatae vel subapiculatae, pedicellos aequantes vel superantes. Sepala membranacea, squamoidea, triangulari-subcordata, breviter acuta, denticulata, mox decidentia, vix $1 \mathrm{~mm}$ longa; petalum superius subcarinatum, ad lobos satis latis denticulato-repandum mucronulatum, ad dorsum non cristatum, sed carina apicem versus subcucullato-geniculata, calcari circiter tertiam partem totius petali $1,1-1,3 \mathrm{~cm}$ longi occupante, decurvato, subbullato-rotundato; petalum inferius subcarinatum, mucronulatum, non cristatum, ad lobos vix denticulato-repandum; petala interiora unguiculata, ungue lineari-oblongo, lamina obliqua, versus petalum superius truncato-lobata. versus inferius paulatim attenuata, vix subpanduraeformis, ad dorsum crista longa, non ita alta, laminae apicem superante, versus basim usque ad unguis basim paulatim decrescente ornata; staminum superiorum appendix nectariferus longus atque rectus. Siliqua patens vel pendula, linearis, subquadrangularis, torulosa, recta vel subarcuata, pedunculo subgracili quam siliqua multo breviore, $2-2,5 \mathrm{~cm}$ longa, stylo satis longo ac gracili coronata; semina $8-12$, minus turgida, ad marginem acutiuscula, splendide atra, modo sub lente levissime reticulata.

Gebiet des pazifischen Nord-Amerikas; Provinz der Rocky Mountains. - Wyoming: Wind River Mts. (W. H. For wood, fl. fr., VII, 1882, no. 139! Typus!). Yellowstone National Park (A. and E. Nelson, Pl. Yellowstone Nat, Park. fl. fr., VII, 1899, no. 5684!).

Nota: Differt ab C. aurea habitu, calcari decurvato, subbullato-rotundato, crista petalorum interiorum usque ad petali basim elongata, seminibus acutiusculis. 
3. Corydalis tortisiliqua Fedde, spec. nov.

Herba perennis glauco-pruinosa, virgulta, e basi multicaulis et valde ramosa, caulibus satis coaretatis, orectis et strictis, pruinosis, valde striato-angulatis, circiter $30 \mathrm{~cm}$ altis. Folia alterna longe petiolata, bipinnatipartita, lobis pinnatilobatis, lobulis, ultimo plerumque trifido, lanceolato-oblongis, breviter acutatis, interdum submucronulatis, petiolis ad basim non ita vaginanter dilatatis. Flores flavidi in racemos terminales atque laterales, multifloros, etiam sub fructibus subconfertos, satis breves, folia non superantes, breviter pedunculatos dispositi; bractea $\theta$ lineari-lanceolatae, longe acutatae, pedicellos aequantes vel paullo superantes. Sepala membranacea, squamoïdea, triangulari-subcordata, breviter acuta, denticulata, permox decidentia, 1,5 mm longa; petalum superius subcarinatum, ad lobos satis latos subrepandum, non mueronulatum, ad dorsum carinae partis subgeniculatae denticulis 4 inter se cohaerentibus obtusiusculis ornatum, calcari paullo plus quam tertiam partem totius petali $1,3-1,4 \mathrm{~cm}$ longi occupante, decurvato, subbullato-rotundato, petalum inferius subcarinatum, subacutum, aequo modo ad dorsum denticulato-cristatum; petala interiora unguiculata, ungue lineari-suboblongo, lamina obliqua, versus petalum superius truncato-lobata, versus inferius modo subitu angustata, vix subpanduraformis, ad dorsum crista longa, dimiduo petali in summa parte alta paulatim usque ad unguis basim decrescente, laminae apicem paullo superante ornata; staminum superiorum appendix nectariferus ad apicem leviter deflexus, satis longus. Siliqua patens vel pendula, linearis, subteres (?), torulosa, subarcuata, pedicello non ita gracili, quam siliqua multo breviore, circiter $2 \mathrm{~cm}$ longa, stylo satis longo et gracili coronata, valvis post dehiscentiam contortis; semina 8-12, minus turgida, ad marginem acutiuscula, splendide atra, modo sub lente levissime reticulata.

Gebiet des pazifischen Nord-Amerikas; Provinz der Rocky Mountains. - Wyoming: Albany Co: Centennial Rambler Road, deep gravel (L. N. Gooding, Pl. Wyoming, fl. fr., IX, 1903, no. 2071!).

Nota: Sine dubio $C$. voyomingensi valde affinis, sed satis distincta habitu virgulto, caulibus satis robustis, compluribus, pruinosis, crista denticulata petalorum exteriorum siliquis post dehiscentiam varie contortis.

4. Corydalis Gooddingii Fedde, spec. nov.

Herba perennis habitu $C$. tortisiliquae valde similis, glauca subvirgulta, e basi multicaulis ot ramosa, caulibus satis robustis, e basi adscendente erectis et strictis; valde striato-angulatis, circiter $25-40 \mathrm{~cm}$ altis. Folia alterna, longe petiolata, bipinnatipartita, lobis pinnatilobatis, fere subflabellatis basim versus cuneatis, lobulis, ultimo stricte trilobulato, lineari-lanceolatis usque ad lanceolato-oblongis, acutatis, intermediis ultimis obovato- vel obcuneato-lanceolatis, breviter acutatis et fere submucronulatis; petiolis basim versus leviter vaginanter dilatatis. Flores flavidi in racemos terminales atque laterales, densifloros atque breves, 
sub fructificatione non multo elongatos, breviter pedunculatos, folia non superantes dispositi; bracteae lineari-lanceolatae, longe acutatae, ad apicem saepius denticulatae quam florum pedicelli usque ad duplo Iongiores. Sepala membranacea, squamoïdea, ovoïdeo-subcordata, longius acuta, denticulata, permox decidentia, 1,5-2 mm longa; petalum superius leviter carinatum, ad lobos satis latos et ad apicem rotundatum subrepandum, non cristatum, calcari paullo plus quam tertiam partem totius petali 1,4-1,6 cm longi occupante, leviter decurvato, subbullatorotundato; petalum inferius levissime carinatum, subacutum, ecristatum; petala interiora unguiculata, ungue lineari-oblongo, lamina obliqua, versus petalum superius truncato-lobata, versus inferius modo paullo contracta, vix subpanduraeformi, ad dorsum crista longa, dimiduo petali in summa parte alta, paulatim usque ad unguis basim decrescente, laminae apicem paullo superante ornata; stamin um superiorum appendix nectariferus leviter subgeniculato-curvatus, satis longus. Siliqua plerumque pendula, lineari-lanceolata, subteres (?), torulosa, subarcuata, pedicello satis crasso, quam siliqua multo breviore, $1,5 \mathrm{~cm}$ longa, stylo satis longo et gracili coronata, valvis post dehiscentiam non contortis, sed ad contortionem vergentibus; semina 9-12, minus turgida, ad marginem acutiuscula, splendide atra, modo sub lente levissime reticulata.

Gebiet des pazifischen Nord-Amerikas; Provinz der Rocky Mountains. - Colorado: Larimer Co., Walden, waste cultivated land (L. N. Gooding, Pl. Colorado, fl. fr., VII, 1903, no. 1501! Exemplar Typicum!). - Utah: Utah Valley (M. E. Jones, Fl. Utah, fl. fr., V, 1880, no. 1682 !).

Nota: Habitu C. tortisiliquae persimilis, differt autem petalis exterioribus cristatis, calcari minus curvato, valvis siliquarum post dehiscentiam non tortuosis, sed modo arcuatis.

\section{Corydalis hypecoiformis Fedde, spec. nov.}

Herba perennis humilis, rosulata, glaucescens habitu Hypecoi procumbentis, e radice crassa perpendiculari uniceps vel multiceps, caulibus diffusis, vel procumbentibus vel adscendentibus vel rariis erectis, angulato-striatis, plerumque $5-7,5 \mathrm{~cm}$, rarius usque ad $10 \mathrm{~cm}$ altis. Folia alterna longepetiolata, subbipinnatipartita, lobis rursus lobulatis vel integris, lobulis lanceolatis vel lineari-lanceolatis, acutis, ut totum folium diminutis; petiolis basin versus distincte vaginanter dilatatis. Flores flavidi in racemos terminales vel laterales, 5-10-floros, satis densifloros et breves, sub fructificatione paullo elongatos, terminales breviter, laterales longe pedunculatos, folia superantes vel aequantes dispositi; bracteae lineari-lanceolatae, longe acutatae, ad apicem denticulatae, quam florum pedicelli pro rata robusti aequilongae vel longiores. Sepala membranacea, squamoïdea, triangulari-ovoïdea, longe acutata satis alte denticulata, decidua, $2-3 \mathrm{~mm}$ longa; petalum superius carinatum, ad lobos repandum, apiculatum, ad dorsum carinae versus apicem levissime cristatum, calcari vix tertiam partem totius petali $1,5-2 \mathrm{~cm}$ 
longi occupante, leviter decurvato, subbullato-rotundato, petalum inferius carinatum, bis subgeniculatum, apiculatum, lobis satis latis grosse denticulatis, ad dorsum leviter cristatum. crista semilunari carinae genui insidente; petala interiora unguiculata, ungue lineari-oblongo, lamina obliqua, versus petalum superius truncato-lobata, versus inferius modo paullo contracta, subpanduraeformi, ad dorsum crista longa. dimiduo petali in summa parte alta, paulatim usque ad unguis basim decrescente, laminae apicem paullo superante ornata; staminum superiorum appendix nectariferus leviter curvatus. Siliqua (immatura) lineari-lanceolata, teres (?), torulosa, arcuata, pedicello satis crasso, quam siliqua multo breviore.

Gebiet des pazifischen Nord-Amerikas; Provinz der Rocky Mountains. - Colorado: Cassels, Platte Canon, 8000-10000 feet (Mrs. A. and Miss Henry, fl. fr., VI, 1894!)

Nota: Habitu humili praeclara et valde affinis $C$. montarae, a qua differt crista etsi minuta petalorum exteriorum, appendice staminum modo leviter curvato, crista petalorum interiorum usque ad basin unguis procedente.

\section{Vermischte neue Diagnosen.}

806. Corydalis hastata Fedde, nom. nov. -- Syn.: Capnoides hastatum Rydberg in Bull. Torr. Bot. Cl., XXXIV (1907), p. 426. - Verwandt mit C. Cusickii S. Wats. - Idaho (C. V. Piper. no. 4057).

807. Corydalis brachycarpa Fedde, nom. nov. - Syn.: Capnoïdes hastatum Rydberg, 1. c., p. 426. - Verwandt mit C. Brandegei S. Wats. - Ltah (M. E. Jones no. 1197; Rydberg no. 6848; Loonard nก. 175).

808. Dicentra occidentalis Fedde, nom. nov. - Syn.: Bicuculla occidentalis Rydberg in Bull. Torr. Bot. Club., XXIX (1902), p. 160. - Nahe verwandt mit $D$. cucullaria. - Washington (Suksdorf).

809. Pandanus IJzermannl Boerl. et Koorders in A. Koorders-Schumacher, System. Verz., II (1910), p. 13. - Arbor 5-7 m alta. Truncus $15-25 \mathrm{~cm}$ diam., erectus, cylindraceus, aculeis brevibus armatus, apice pauce ramosus, basi radicibus aëreis suffultus. Folia $1-3 \mathrm{~m}$ longa et $6 \mathrm{~cm}$ lata, in acumen breve triquetrum desinentia, margine et costae supra dense spinuloso-serrata, spinulis minimis acutis strictis. Syncarpia magna, rubra. - Die Blüten und Früchte sind durch den ausserordentlich schwierigen Transport quer durch den überschwemmten Wald verloren gegangen. Die Blätter erinnern an Pandanus caricosus S. Kurz, aber unsere Art ist u. a. durch den baumartigen Habitus und die viel grösseren Syncarpien deutlich verschieden. - Mittel-Sumatra: Kds. 10400 B. - 